\title{
Asia a través de la Academia Occidental: un análisis comparativo
}

\section{Asia through the Western Academy: a comparative analysis}

Santiago Carranco Paredes

Universidad Internacional del Ecuador, Ecuador

Autor para correspondencia: scarrancoparedes@gmail.com, sacarrancopa@internacional.edu.ec Fecha de recepción: 10 de Junio de 2017 - Fecha de aceptación: 30 de Septiembre de 2017

\section{Resumen \\ La idea de que Asia y los estudios sobre este continente deben tener una gran preeminencia para la academia latinoamericana y noroccidental se ha convertido en un cliché. Los estudios que han confirmado la preponderancia de estados asiáticos para la comprensión de las relaciones comerciales, sociales, políticas, económicas y académicas actuales, tanto a un nivel global como regional, son vastos. El presente estudio no se va a preocupar por evidenciar la expansión de la influencia asiática dentro de la academia latinoamericana, más bien, se limitará a reseñar el modo en el que las universidades latinoamericanas y andinas están estudiando al sudeste asiático en comparación con las universidades de la región Noroccidental.}

Palabras Clave: academia; internacionalización; asia; relaciones internacionales; estudios internacionales

\begin{abstract}
The conception about the importance of the Asian Studies inside the Latin American and Northwestern academy is something obvious in these days. Several studies have confirmed the preponderance that the Asian states has, to understand the current commercial, social, political, economic, and academic relations in the global arena. The present study is not going to concern itself with showing the expansion of Asian influence within the Latin American academy, rather, it will be limited to outlining the way in which Latin American and Andean universities are studying Southeast Asia in comparison to the universities of the North-western region.
\end{abstract}

Key words: academy; internationalization; asia; international relations; international studies 


\section{Introducción}

Desde la década de los ochenta del siglo pasado, se ha venido produciendo una desconcentración de poder en el sistema global. La sociedad internacional se ha visto afectada por un desplazamiento continuo del centro del poder geopolítico desde el Noroccidente hacia la región de Asia-Pacífico; un cambio que, si en un primer momento fue económico, progresivamente se ha ido convirtiendo también en político y cultural. De esta manera, mientras los mercados emergentes asiáticos han ido ascendiendo en la cadena de valor de los procesos de producción industrial, el impacto de los países industrializados noroccidentales en relación a su proporción relativa del PIB mundial ha ido disminuyendo. Para El Aynaoui \& Woertz 2016:7-8, esta nueva industrialización, no solo ha impactado en los indicadores económicos que expresan las fluctuaciones en el comercio global, sino que ha afectado el desarrollo propio de las sociedades occidentales. Es así que Asia ha sido capaz de desarrollar "Soft Power" y cierta hegemonía cultural propia, ya sea en el ámbito de la cultura pop, la filosofía, el mundo académico o la arquitectura; así lo demuestra el éxito del conocido "Gangnam Style", las improvisadas salas de yoga en los gimnasios tradicionales, la ola de inauguraciones de restaurantes de cocina asiática, los diseños urbanos futuristas y la creación de un ranking propio de las universidades del mundo (ARWU, por sus siglas en inglés) elaborado por la Universidad Jiao Tong de Shanghái, en donde existen más de 100 universidades del sudeste asiático dentro del top500 a nivel mundial (ShanghaiRanking Consultansy 2016).

La idea de que Asia y los estudios sobre este continente deben tener una gran preeminencia para la academia latinoamericana y noroccidental se ha convertido en un cliché. Los estudios que han confirmado la preponderancia de estados asiáticos como China, Japón, Corea del Sur, India entre otros para la comprensión de las relaciones comerciales, sociales, políticas, económicas y académicas actuales, tanto a un nivel global como regional, son vastos. De igual manera, la evidencia empírica confirma el papel preponderante que representa el Sudeste asiático en la configuración de la sociedad internacional contemporánea, por lo que en la actualidad se hace inconcebible en el estudio académico de Relaciones Internacionales dejar de lado al continente asiático (Kohno 2014) (Bisley 2011).

En la última década, la academia latinoamericana exhibió una fascinación por el crecimiento de China y su creciente influencia en esta región. Un número considerable de autores latinoamericanos se dedicó a entender la dinámica de estas nacientes relaciones entre ambos continentes, entre ellos Manríquez (2014), Álvarez (2014), Panizza (2008), entre otros. Esto motivo a que exista una gran producción regional sobre el tema, lo cual llevó a que varias editoriales regionales dediquen espacios exclusivos para artículos relacionados con China o con el Sudeste Asiático. Un ejemplo de esto es el caso de la Revista Andina de Estudios Políticos que en 2016 publicó un Dossier titulado "China y América Latina: industrias extractivas, política exterior e integración". Por su parte, la Revista Estudios de la Universidad de Costa Rica publicó en el mismo año un Dossier exclusivamente para tratar 'El Estudio sobre China desde (Latino) América"'. 


\section{Metodología}

El presente estudio no se va a preocupar por evidenciar la expansión de la influencia asiática dentro de la academia latinoamericana, más bien, se limitará a reseñar el modo en el que las universidades latinoamericanas y andinas están estudiando al sudeste asiático en comparación con las universidades de la región Noroccidental. Para el estudio se ejecutó un análisis estadístico de codependencia de variables cualitativas a partir de una muestra de las mejores universidades de cada región, obtenidas del Ranking Web de Universidades (Webometric). De este ranking se tomó a las 10 mejores universidades de América Latina y de la región Andina ${ }^{1}$, para compararlas con las mejores 50 universidades a nivel mundial, en su totalidad provenientes de la región noroccidental. A cada una de ellas se les atribuyó un número de variables relacionadas con el estudio de Asia en el campo de las Relaciones Internacionales y de los Negocios Internacionales, ya que como se lo mencionó previamente, estas son las carreras que tendrían la necesidad de afrontar académicamente el fenómeno de la expansión de la influencia asiática hacia el mundo occidental. Para el análisis de las variables se utilizó el estadístico Chicuadrado, el cual permite evaluar la relación de dependencia entre las variables seleccionadas. Es así como se logró llegar a una percepción general sobre la manera en la que la academia de las tres regiones está incluyendo el estudio de Asia dentro de sus mallas curriculares en las materias de Negocios Internacionales y de Relaciones Internacionales.

Adicionalmente, se analizó la percepción de los estudiantes de la región andina sobre Asia, por lo que se eligió una muestra de estudiantes de las Carreras de Relaciones Internacionales y Negocios Internacionales de las universidades de la Región Andina que cuenten con dos características principales, la primera es que hayan cursado una materia relacionada con Asia en su pregrado, y la segunda que hayan realizado algún tipo de curso académico en la región del Sudeste asiático. De esta manera, se pudo definir no solo la percepción general de la situación de las universidades respecto a su estudio de Asia, sino también el tipo de información que se difunde dentro de las clases relacionadas con dicho continente.

El análisis de la muestra se la dividió en tres partes correspondiente a cada una de las regiones de América Latina, Región Andina y Noroccidente, a fin de poder contrastar el estado de los estudios asiáticos dentro de las carreras de Relaciones Internacionales y Negocios internacionales en las tres regiones. A su vez, se estableció categorías sobre las materias relacionadas con Asia con el objetivo de homologar materias similares bajo una misma variable, reduciendo el número de materias a cuatro ítems: Estudios Asiáticos, Estudios Afroasiáticos, Relaciones Internacionales de Asia y Asia-Pacífico. En los gráficos que se presentan a continuación se puede apreciar estas distinciones.

\footnotetext{
${ }^{1}$ Los datos de las universidades venezolanas se tomaron de páginas privadas y de universidades que disponen de información, al encontrar que las páginas de universidades públicas tienen una restricción electrónica internacional.
} 


\section{Análisis de Resultados}

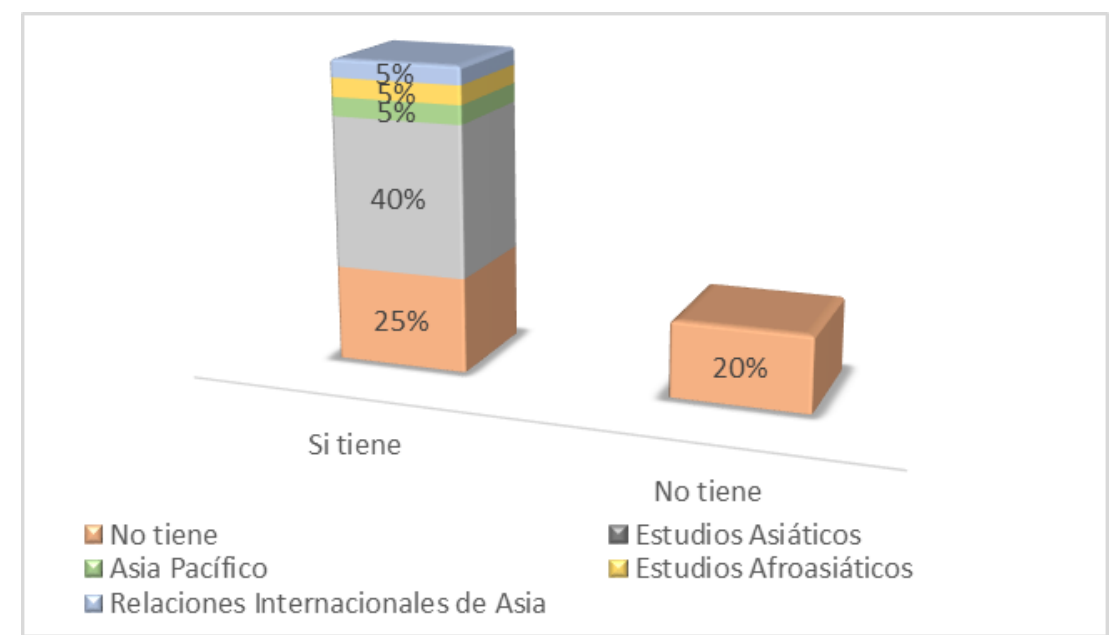

Gráfico 1: Carrera de Relaciones Internacionales en Latinoamérica Elaborado Por el Autor

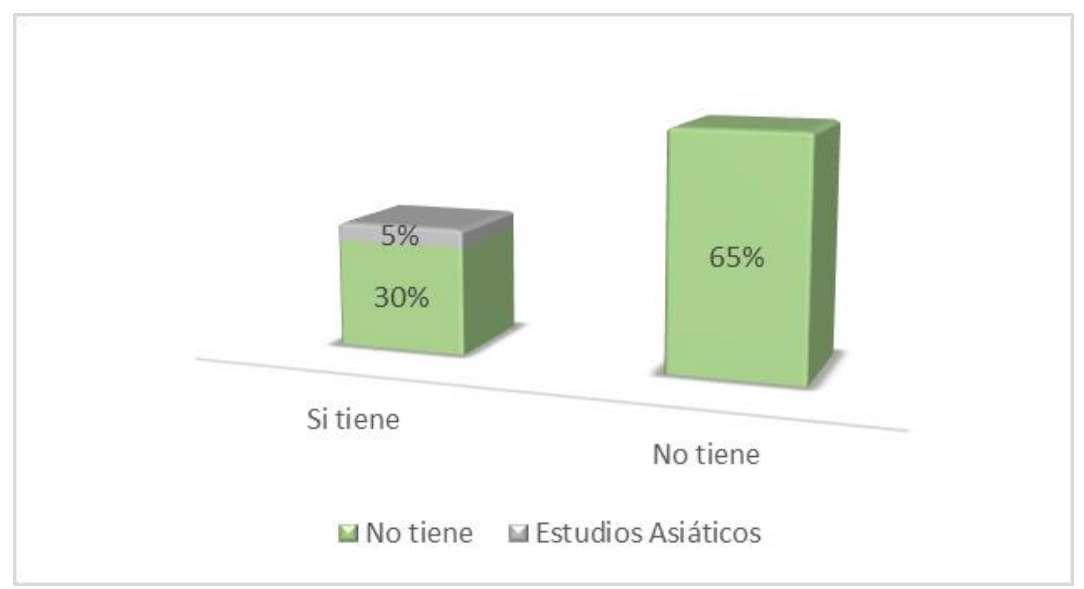

Gráfico 2: Carrera de Negocios Internacionales en Latinoamérica Elaborado Por el Autor

En el caso de América Latina se puede observar en el Gráfico 1 que el estudio de relaciones internacionales está presente en la mayor parte de universidades de la región, y que además las materias relacionadas con el continente asiático están presentes de manera general. Se evidencia que el 15\% de universidades de la muestra tienen materias específicas sobre Asia, incluyendo materias relacionadas con Asia Pacífico y Relaciones Internacionales de Asia. En lo que respeta al análisis de Asia dentro de los estudios de Negocios Internacionales de la región, éste no es preponderante. Hu-DeHart (2010:236-238), explica que durante la década de los 80 y 90 la academia brasilera, argentina y chilena se enfocó en estudiar los flujos migratorios que se dieron desde Asia y el Medio Oriente hacia América del Sur luego de la segunda guerra mundial, lo cual originó una especie de costumbre académica multicultural. Adicionalmente, era común ver estudiantes de origen asiático cursando sus estudios especialmente en universidades brasileras y argentinas, por lo que el interés en los estudios asiáticos se fue ganando un espacio dentro de la academia en esta región (Hu-DeHart 2010 241-242) (Ko 2016). En este sentido, se 
puede concebir que el estudio sobre flujos migratorios es lo que catapultó a los estudios asiáticos a tener un papel importante dentro de esta región, es claro que siendo un tema concerniente a Política Internacional sea justamente las escuelas de Relaciones Internacionales la que lo aborden.

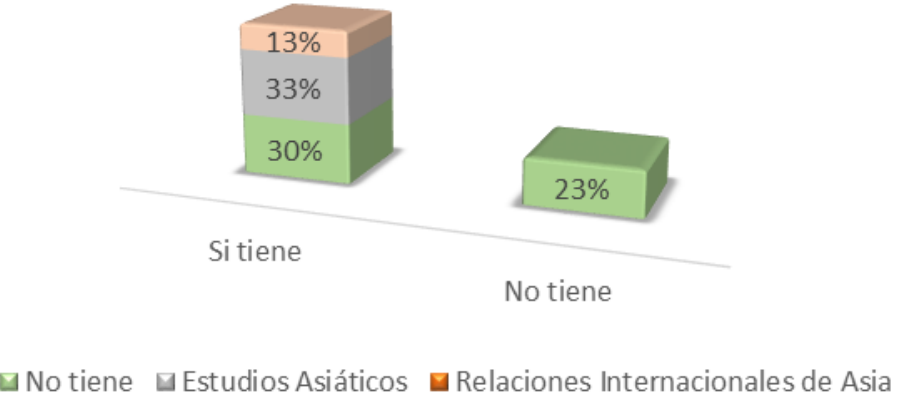

Gráfico 3: Carreras de Relaciones Internacionales en el Noroccidente Elaborado Por el Autor

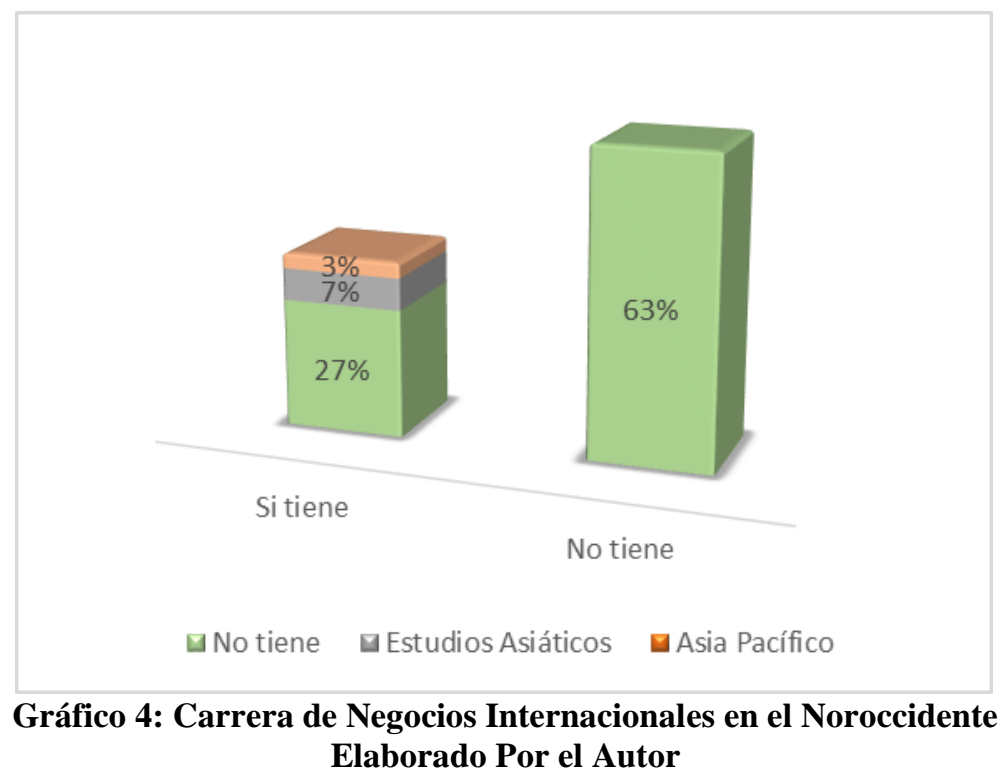

El Gráfico 3, el cual es concerniente a la academia noroccidental evidencia la importancia que tienen los estudios asiáticos dentro de la materia de relaciones internacionales, impartidas en estos países. Sin embargo, al analizar la materia de negocios internacionales, ésta ha quedado relegada, sin revisar los estudios sobre Asia.

La estrecha relación entre escuelas de Relaciones Internacionales y los estudios asiáticos de la región noroccidental, viene dada por una larga tradición histórica fundada en las relaciones comerciales, además de los flujos migratorios. Durkin (2008), propone el caso de la academia inglesa, en donde demuestra el creciente número de estudiantes de la región asiática desde la década de los ochenta. En este caso se demuestra como la cantidad masiva de estudiantes asiáticos ha reconfigurado la educación en el Reino Unido. De esta manera, se entiende que el 
crecimiento económico en los países del sudeste Asia se ha reflejado en el mejoramiento de políticas educacionales, en donde los gobiernos han impulsado el envío de sus jóvenes a academias de estados industrializados para prepararse, como parte de un programa de industrialización y transferencia de conocimiento (Durkin 2008).

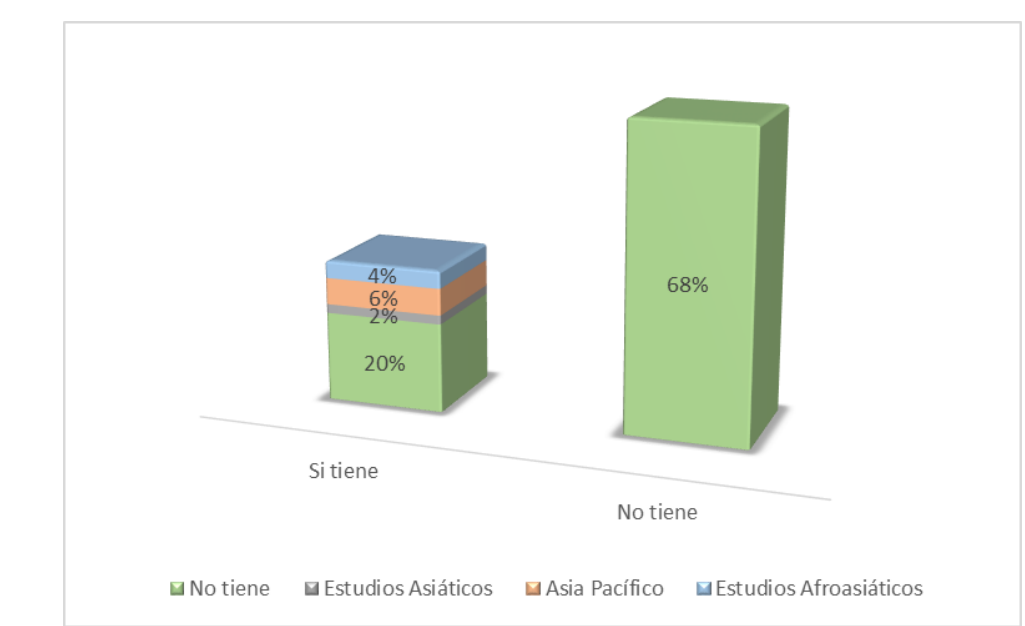

\section{Gráfico 5: Carrera de Relaciones Internacionales en la Región Andina Elaborado Por el Autor}

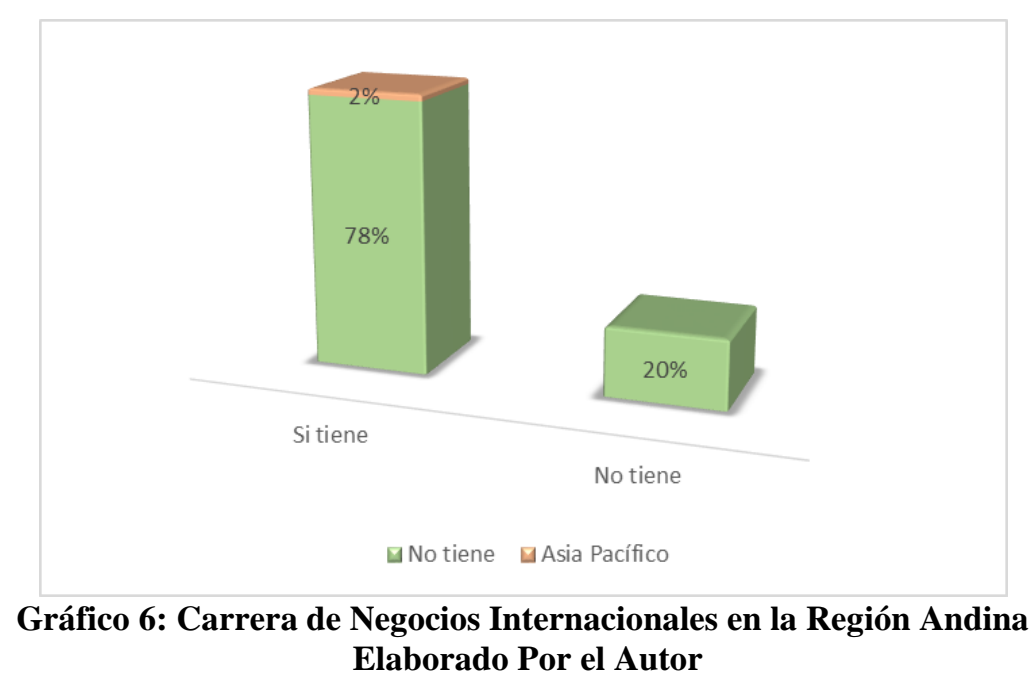

En lo que respecta a la región Andina se vislumbra que existe un menor porcentaje de escuelas de Relaciones Internacionales en comparación con Latinoamérica y con el Noroccidente, a su vez, el interés sobre estudios asiáticos aún no ha despertado, algo que es corroborado al observar las respuestas de los alumnos andinos que han migrado a Asia. Exponiendo una síntesis de sus respuestas se puede deducir que su interés sobre los estudios asiáticos no nace en su pregrado, sino que este nace desde la necesidad profesional. La presencia de los estados asiáticos, en especial de China en el territorio Andino, se ha extendido en los últimos años, y queda claro que existe un vacío dentro de los estudiantes que salen de la academia sobre las cuestiones referentes a Asia, por lo que los estudiantes tratan de llenarlo a partir de una búsqueda ya sea de maestrías o cursos de verano en universidades asiáticas. 
La importancia de Asia para el mundo es incuestionable, es claro que este ha pasado de ser el continente místico y desconocido para el occidente, como lo proponía Edward Sid (BeitHallahmi 1980), a ser un objeto de estudio de la academia occidental, como se demuestra en los casos de academia noroccidental y latinoamericana. Por otro lado, Asia ha despertado y ahora se ha transformando en el productor de conocimiento. El ranking de universidades de Shanghái es una prueba de la fragmentación y de la autonomía que propone la academia asiática hacia la región occidental.

Cuando se le preguntó a una estudiante andina que cursa su maestría en la Universidad Politécnica de Hong Kong nos indicó que:

"El sistema educativo es uno de los contrastes más significativos entre América latina y Asia. La metodología y el pensamiento de la academia oriental, limitándome específicamente a China, están muy fundamentados en la doctrina de Confucio. En tal sentido, el enfoque siempre será de una tendencia colectivista en el cual prevalecen los intereses globales sobre los particulares. "(Carillo, 2017)

Es importante destacar que el rápido crecimiento económico de China e India, y el desarrollo que se ha producido en los estados del Sudeste Asiático han atraído la atención de académicos e investigadores, y ha tenido un gran impacto en el campo de los estudios asiáticos. Esto a su vez ha producido un cambio en la percepción que se tiene de Asia no como un objeto de estudio, sino como un productor de conocimiento (Winichakul 2014).

\section{Conclusiones}

Luego del término de la Guerra Fría, la academia tuvo que exponerse a varios procesos de cambio para poder adaptarse a esta nueva era de globalización dentro del sistema unipolar. Por esta razón, en los últimos años la idea de la internacionalización de la academia se ha convertido en un tema preponderante para la las universidades a nivel mundial (Winichakul 2014:881). Este pensamiento de la internacionalización ha abierto un campo interesante para que estudiantes occidentales tengan acceso al conocimiento de Asia. No obstante, al hacer una comparación entre la región Andina, Latinoamérica y el Noroccidente, nos damos cuenta de que hay diferentes grados de internacionalización en cada una de estas regiones. Por un lado, el noroccidente mantiene una educación especializada y multicultural en la que no sólo se estudia a Asia, sino que se aprende de ella. Por su parte, en América Latina se ha logrado institucionalizar a Asia como un objeto de estudio mediante la diversidad en sus mallas curriculares. Mientras que la región Andina sufre un retraso en la incorporación de estudios asiáticos a sus mallas curriculares, por lo que los estudiantes interesados en Asia han tenido que recurrir a vías alternas para poder acceder a dicho conocimiento.

\section{Bibliografía}

Beit-Hallahmi, Benjamin 1980 Review of Orientalism. Journal for the Scientific Study of Religion 19(1): 69-70. 
Bisley, Nick 2011 Asia's Transformation, International Relations and Public Policy. Australian Journal of International Affairs 65(1): 102-108.

Durkin, Kathy 2008 The Middle Way: East Asian Master's Students' Perceptions of Critical Argumentation in U.K. Universities. Journal of Studies in International Education 12(1): $38-55$.

El Aynaoui, Karim, and Eckart Woertz 2016 Introducción: África, América Latina y El «siglo de Asia». Revista CIDOB d'afers Internacionals(114): 7-16.

Hu-DeHart, Evelyn 2010 Multiculturalism in Latin American Studies: Locating the "Asian" Immigrant; or, Where Are the Chinos and Turcos? Latin American Research Review 44(2): $235-242$.

Ko, Chisu Teresa 2016 Toward Asian Argentine Studies. Latin American Research Review 51(4): 271-289.

Kohno, Masaru 2014 East Asia and International Relations Theory. International Relations of the Asia-Pacific 14(1): 179-190.

ShanghaiRanking Consultansy 2016 Discovering World Class. https://doc-0k-aodocs.googleusercontent.com/docs/securesc/kffmvot8g48hsb9807ci97cds8c3tqu0/hj9v9d6 jid5eu5k3ead8q2o4oq561533/1496419200000/08619350468429608082/08334199373923 561037/0Bw2rAawlHlvBSFZiVnBNZUFCVDA?e=download\&nonce=8bcm891 da9t4s \& user $=08334199373923561037 \&$ hash=ihka660f0spm9samua3qbc2opnjfhieb, accessed June 2, 2017.

Winichakul, Thongchai 2014Asian Studies across Academies. The Journal of Asian Studies 73(04): 879-897. 